\title{
THE USE OF TOROIDAL BOUNDARY CONDITIONS IN THE PROGRAM POISSON*
}

\author{
S. Caspi, M. Helm, and L. J. Laslett
}

\author{
Lawrence Berkeley Laboratory \\ University of California \\ Berkeley, CA. 94720
}

September, 1987

\section{DISCLAIMER}

\begin{abstract}
This report was prepared as an account of work sponsored by an agency of the United States Government. Neither the United States Government nor any agency thereof, nor any of their employees, makes any warranty, express or implied, or assumes any legal liability or responsibility for the accuracy, completeness, or isefulness of any information, apparatus, product, or process disclosed, or represents that its use would not infringe privately owned rights. Reference herein to any specific commercial product, process, or service by trade name, trademark, manufacturer, or otherwise does not necessarily constitute or imply its endorsement, recommendation, or favoring by the United States Government or any agency thereof. The views and opinions of authors expressed herein do not necessarily state or reflect those of the United States Government or any agency thereof.
\end{abstract}

* This work was supported by the Director, Office of Energy Research, Office of High Energy and Nuclear Physics, High Energy Physics Division, U.S. Dept. of Energy, under Contract No. DE-ACD3-76SF00098. 
S. Caspi, M. Helm, and L. J. Laslett

Lawrence Berkeley Laboratory

University of Californla

Berkeley, CA 94720 , USA

\section{Abstrack}

In circular particle accelerators of moderate size, one cannot entirely neglect the curvature of the structure and of the gulde fleld. in practice, one may wish to restrict the region of analysis to that near the working aperture, while excludling a very substantlal area closer to (and including) the axis of rotational symmetry. in this way. a more efflcient mesh can be generated for a program such as POISSON. In restricting the solution to the region of interest, there must be concern regarding a suitable termination of the problem at the boundary of the mesh. For these reasons, we have employed toroidal coodinates in constructing the bourdary to a relaxation mesh, and in formulating the boundary conditions that then would be Imposed at such boundaries.

\section{Introduction}

This naper is an extension of a series of papers and reports on the use of boundary conditions associated with application of relaxation methods in the solution of partial diffetentlal equations.2-20 The curtent paper is a summary of one such reportse on the use of such boundary conditlons in problems with axial symmetry.

Magnetostatlc problems with circular symmetry are soluble by relaxation programs such as POISSON in $p, Z$ cylindrical coordinates. As is the case with other applleatlons of relaxation methods, however, there must be concern regarding a sultable termifiation of the problem at the boundary of the mesh. (The condition that normally is required ls one conslstent with the absence of any "sources" in the reglon exterlor to such a boundary.) in analyzing the magnetic fields of circular partlcle accelerators, one may wish to restrlct the region of examination to that near the working aperture and surrounding magnet structure, while excluding a very substantlal area closer to (and including) the axis of rotational symmetry for the entire structure.

For the reason just indicated, one accordingly is led to consider the use of toroidal coordinates in constructing the boundary to a relaxation mesh for use in analyzing the magnetic fields of circular devices (ach as accelerators and spectrometers), and in formulating the boundary conditions that then may be usefully Imposed at such boundaries. The procedure adopted maikes use internally of the characteristic form of the vector-potential function, in a source-free region, when expressed in toroidal coordinates. The relevant properties of associated Legendre functions of half-Integral degree are used in this comection and their Introduction into the program POISSON is cutlined. Results of some test cases are included, to illustrate the application of thls technique for conflqurations with median-plane symmetry. We pursue such issues in the following Sections, commenclng with a review of the characteristics of Loroidal coordinates and continuing with an examination of related magnetostatle issues that will permit formulation of a boundary condition analogous in spirit to those devised previously at this Laboratory for application to other configurations. $1 \rightarrow$

This work was supported by the Director, Office of Energy Research, Office of Hlgh Energy and Nuclear Physics, High Energy Physics Division, U.S. Dept. of Energy, under Contract No. DE-ACD3-765F00098.

\section{The Differential Eorntion for the Potential}

Through use of the appropriate metric coefficients, one can write explicitly in toroidal coordinates Laplace's differential equation for a scalar potential function. As has been shown in some detall by MacRobert, 22 solutions then may be found in which this potential function has the form

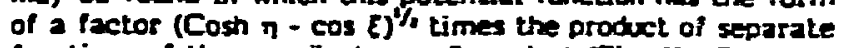
functions of the coordinates $n, E$. and $\$$ (Fig. 1). For such solutions, the functions of $E$ and of $\phi$ are each just circular functions of their respective arguments, and the functions of $\eta$ are Legendre functions (or associated Legendre functions) of half-integral degree and argument $z=$ Cosh $n$

In the present work, however, we are specifically Interested in POlsSON computations of masnetic field for cases with axial symmetry and wish to make use of a vector-potential component $A_{\phi}$ (or $A^{\circ}=\rho A_{\phi}$ ) to characterize this field. The homogeneous equation $\nabla \times[\nabla \times \vec{A}]=0$ for $A=A_{\phi} C_{\phi}$ then may be written

$$
\begin{aligned}
& \frac{\partial}{\partial \eta}\left[\frac{\cosh n-\cos \varepsilon}{\sinh n} \frac{\partial}{\partial n}\left(\frac{\sinh n}{\operatorname{Cosh} n-\cos E} A_{\phi}\right)\right] \\
+ & \frac{\partial}{\partial \xi}\left[\frac{\cosh n-\cos \varepsilon}{\sinh n} \frac{\partial}{\partial \varepsilon}\left(\frac{\sinh n}{\cosh n-\cos E} A_{\phi}\right)\right]=0
\end{aligned}
$$

- - wherein the dependent variable $A_{4}$ is to be regarded as a function of $\eta$ and $E$, but independent of $\phi$.

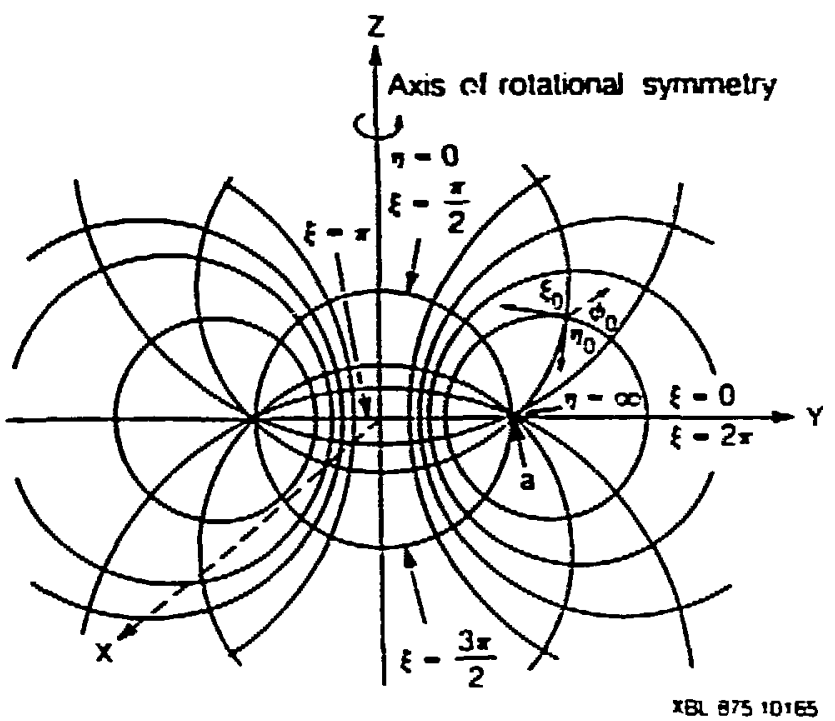

Figure 1. Toroidal Coordinates $(\varepsilon, \eta, \phi)$

Cuided by the form known to be appropriate for the scalar-potential solutions to Laplace's equation in toroidal coordinates, we mar proceed heuristically to achieve a separation of variables in the present case, once $A_{\phi}$ is divided by the factor $(\operatorname{Cosh} \eta-\cos E)^{1 / 4}$. We accordingly write the vector-potential component. $A_{\phi}$ in the form

$$
A_{\phi}=(\operatorname{Cosh} \pi-\cos E)^{2 / 2} \cos n \xi C(\pi) .
$$


With thls subatltution, the differential equation assumes the form

$$
\frac{d}{d z}\left[\left(z^{2}-1\right) \frac{d C}{d z}\right] \cdot\left[\frac{1}{z^{2}-1}+v(u+1)\right] c=0 \text {, }
$$

(following some intermediate algebraic work), the $\xi$ dependence then is found to disappear (as hoped), and there remains only the ordinary differential equation for the factor $G(z)$ itsalf with $z=$ Cosh $n$ serving as the independent varimble (and $0=n-1 / 2$ ). Solutions to the differential equation for $\mathbf{C}$ can be written as directly proportional to associated $(m=1)$ Legendre functions of degree $v=n-1 / 2$ and argument $z=$ Cosh $n$. We shall employ in the work to follow only the functions of the first kind, $P(k=n-1 / 2(z)$ or quantitles proportional thereto, in order to avoid singularitles developing at remote locations (as the argument $z$ aporazches unity from above). With the index $n$ confined to integer values (to insure a single-valued dependence upon the coordinate E), we thus are confined to terms of the formt

For $A_{\phi}:(\operatorname{Cosh} n-\cos \xi)^{1 / 6} P_{v=\pi-1 / 2}^{2}(2) \cos n \xi$

or

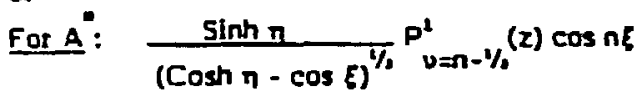

that contain as factors Legendre functions of half-integral degree $(v=-1 / 2,1 / 2,3 / 2, \ldots)$ and which we choose to be even about the mid-plane $\varepsilon=0$.

\section{Apolication}

The proposed boundary condition is illustrated by a boundary so located that no external sources are present The vector potential function external to that boundary is therefore expressible as a summation of terms of the form of Eq. I. If the boundary is conveniently placed on a curve (surface) on which the toroidal coordinate $\eta$ has a constant value $\eta=\eta_{i n}$, the Legendre functions represent a series of harmonic coefficlents. If. In practice, values of the potential are known at only a finite number of points on the "inner boundary" then, of course, only a finite number of harmonic coefficients could be evaluated. such a serles may, however, be adapted to provide adequate estimates of the corresponding values of the potential at varlous polnts on a nearby surrounding "outer boundary" curve, with $n=$ rout.

In performing a relaxation computation on a mesh bounded by such a pair of curves (external to all "sources"). any full relaxation pass through the mesh may be followed by a step wherein the values of potential at points on the outer boundary are revised (updated) on the basis of a harmonic description of the potential function on the innes curve. Such revised values would then be employed, as boundary values, In proceeding with the next relaxation pass through the mesh.

In application, we shall use the forms for $A^{\circ}=\rho A^{\prime}$ to guide the means of extending this function from an "Iriter" boundary curve, $n=\pi_{3 n}$, to points on a surrounding "outerboundary curve. This "outer", or surrounding, boundary curve may conveniently be taken also to be a curve (surface) on which the coroidal coordinate $\eta$ has a constant value ( $n=$ Tout).

It appears compulationally desirable, however, to regard the function $A$ as represented not in the form of a seriss that contaim as explicit factors the Legendre

tOnly the use of a factor cos ne (in preference to a factor $\sin n \xi$ ) is indicated here, since we shall ultimately wish to spectalize to cases with median-plane symmetry such that the function $A_{\phi}$ is even with respect to the variable $E$. functions $P_{p=n-y / s}^{\prime}(x)$, but that introduces in theit place factors ASP $_{2}(z)$ that represent such functions renormalized through division by the asymptotic form for $P t$, to provide the working functions for computational use.

To specify in toroidal coordinates a suitable inner boundary. for POISSDN computation of a magnetostatic problem with rotational symmetry, we first select a suitable region of interest in $\rho, Z$ soace such that one is assured that there are no "sources" exterior to this region.

We may imagine the values of the provisional vector potential on the boundary curve $\pi_{\text {in }}$ to be developed in the form of a Fourier series for the working variable $A=\rho A_{\phi}$

$$
\dot{A}\left(n_{\text {in }}, E\right)=\frac{\sinh n_{\text {in }}}{\sqrt{\operatorname{Cosh} n_{\text {in }}-\cos \xi}} \sum_{k=1} C_{k} \cos (k-1) E
$$

(for situatlons of even symmetry, with respect to $E_{\text {, about } z} \mathbf{= 0}$ ).

Civen values of the function $A^{*}\left(\pi_{i n}, \xi_{i}\right)$ for points $\xi_{i}$ on the


(with weights $w_{i}$ ) so as to minimize

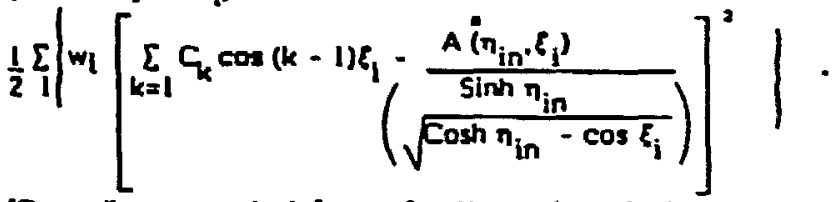

[Regarding augested forms for the weight factors $w_{i}$, see the section included on p.5 of Ref. 5 pertaining to weights ured in comeation with circular functions $F(v)$.]

This minimization objective leads to the set of algebraic equblons that can be written, in matrix notation, $\left[M_{k, 2} C_{l}=V_{k}\right.$. where $M$ is the symmetric matrix with k, 2 elements

$$
\begin{aligned}
M_{k, 2}=\sum w_{1} \cos (k-1) \varepsilon_{i} \cos (2-1) \varepsilon_{i} \\
\text { and } \left.\quad v_{k}=\sum w_{i} \cos (k-1) \xi_{i} \cdot \frac{A\left(\eta_{i n} \cdot \xi_{i}\right)}{\frac{\sinh \eta_{i n}}{\sqrt{\cosh n_{i n}-\cos \xi_{i}}}}\right)
\end{aligned}
$$

Accordingly, the solution may be written in terms of the elements of the inverse matrix, as $C_{\ell}=\sum\left(M^{-1}\right)_{2, k} V_{k}$.

With substitution of the expression for $C_{2}$, there results the working equation (for use in updating values of $A$ on the outer boundary):

$$
A\left(\eta_{\text {out }} \cdot E_{j}\right)=\sum_{l} E_{j, 1} \dot{A}^{\bullet}\left(\eta_{\text {in, }} E_{l}\right) .
$$

where the "working matrix" (a rectangular matrix) is composed of the elements

$$
\begin{aligned}
& E_{j, 1}=\frac{\sinh n_{\text {out }}}{\sqrt{\operatorname{Cosh} n_{\text {out }}-\cos \varepsilon_{j}}} \cdot \frac{\sqrt{\cosh n_{\text {in }}-\cos \varepsilon_{i}}}{\sinh \eta_{\text {in }}}
\end{aligned}
$$

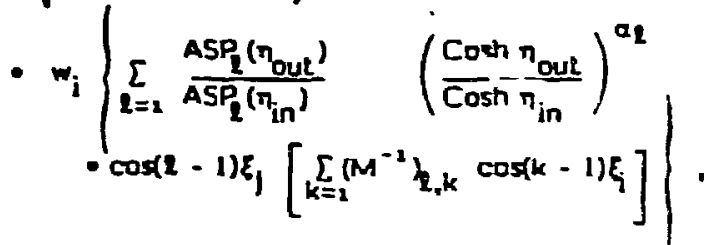


and where $a_{l}=\left\{\begin{array}{l}0 \text { for } i=1 \\ \ell-, / 2 \text { for } 122 .\end{array}\right.$

\section{Introducing The Boundaries Into POISSON'S Mesh Generator}

The use of the toroidal coordinate system in solving problems with axial symmetry requires an eccentric pair of circular ares at the tuundary of such a problem (1.e., no external sources are permitted). The specification for the center and radlus of one of the arcs is a matter of choice; these values are then used to compute the center and radius of the other arc, using the procedure described betow (Flg. 2a).
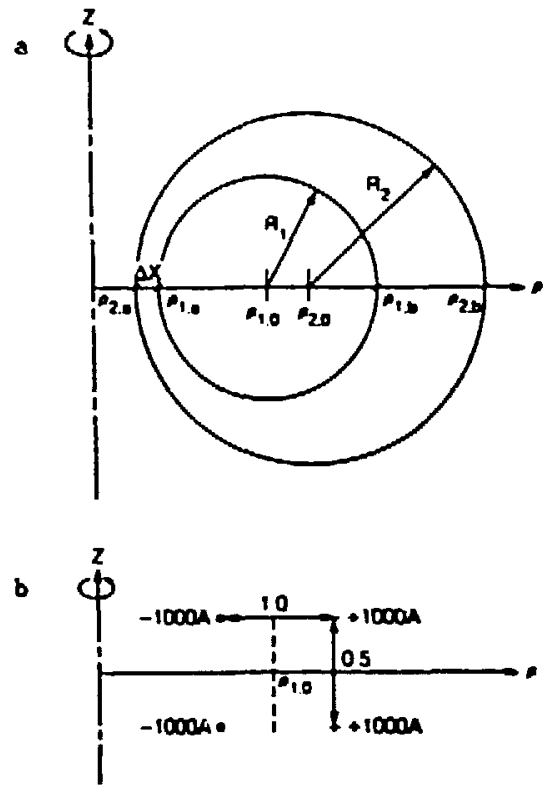

X8L 875-10166

Flg. 2. (a) The Inner and outer boundary used with the toroldal coordlnate system. (b) Location of the a current loops used in the example.

We have chosen to asaign values for $\rho_{2,0}$ and $R_{2}$ (center and radlus) of the inner boundary and compute the corresponding values, $p_{2,0}$ and $R_{2}$ of the outer boundary. (The values of $\rho_{2,0}$ and $R_{1}$ are arbitrary as long as there are no sources outside $R_{2}$ )

Once $F_{1}$ and $p_{1,0}$ are known, we calculate the focal length $a ; a^{2}=0 \%, 0-R_{1}^{2}$.

The minor intersection point between a circular boundary and the abscissa is a - $\operatorname{Tanh}\left(\frac{1}{2}\right)$. The cistance $\Delta x$ (Flg. 2a) between two such boundaries on the absissa Is:

$$
\Delta x=\rho_{1,0}-R_{2}-a \cdot \operatorname{Tanh}\left(\frac{n_{\text {out }}}{2}\right) \text {. }
$$

Assuming that $\Delta x$ is assigned, we calculate nout:

$$
\begin{aligned}
\pi_{\text {out }} & =2 \operatorname{Tanh}^{-2}\left[\frac{\rho_{1,0}-\left(R_{1}+\Delta x\right)}{a}\right] \\
& =\ln \left[\frac{a+p_{1,0}-(R+\Delta x)}{a-p_{1,0}+\left(R_{1}+\Delta x\right)}\right] .
\end{aligned}
$$

We can now calculate the center and radius of the outer boundary:

$$
p_{2,0}=\frac{a}{\operatorname{Tanh} n_{\text {out }}} ; R_{2}=\frac{a}{\sinh n_{\text {out }}} .
$$

When the mesh generator to the program POISSON is used to generate such boundaries, $\Delta x$ can be set to the nominal grid spacing. Thls will assure the existence of a finite dlstance between the boundaries and prevent them from collapsing into each other. It is, however, advisable to Increase the mesh dansity at thls point, which can be easily done by choosing a $\Delta x$ that is larger by an integer multiple of the nominal grid spacing.

\section{Example}

To demonstrate the use of the toroidal boundary condition, we have used a set of coils in a configuration shown in Fig. $2 b$. We have placed $1000 \mathrm{~A}$ in each coil in the Indicated directions and computed $A=P A$ vs. $P$ at $z=0$. We further computed $B_{2}$ along that same path and $B_{r}$ vs. $z$ at the mid radius between the two coils. In addition, the same functions have been computed analytically for both conventional axlsyinmetric and cartesian geometrles. The above computations were done at an increasing focal dimension (parameter a); however, the relative position of the coils, with respect to each other and to the mesh boundaries, remained unchanged. (In all problems a midplane symmetry is assured by specification of a Neumann boundary condltion for $A$ at $z=0$, and the relaxation computations were then performed only in the region $2 \geq 0$.)

\section{Case A - Coils Close to the Axis}

The colls were placed at $\rho_{2}=3.25 \mathrm{~cm}(-1000 \mathrm{~A}), p_{p}=$ $4.25 \mathrm{~cm}(+1000$ A), with each at $z=0.25 \mathrm{~cm}$. The inher boundary was centered midway between the coils at $p_{10}=$ 3.75, with a radius of $R_{1}=1.25$. We assumed $\Delta x=0.1$ and computed nout $=1.65385404$ rad., 0 that $p_{2,0}=3.8042$ and $R_{3}=1.4042$

The close proximity of the coils to the axis of symmetry in this example permitted a solution that lncludes the axis of symmetry and a circular type boundary condition. Flux plots for a toroldal b.c. and a circular b.c. are shown in Flg. 3. Variations in $A$ are compared



XBi. $875-2363$

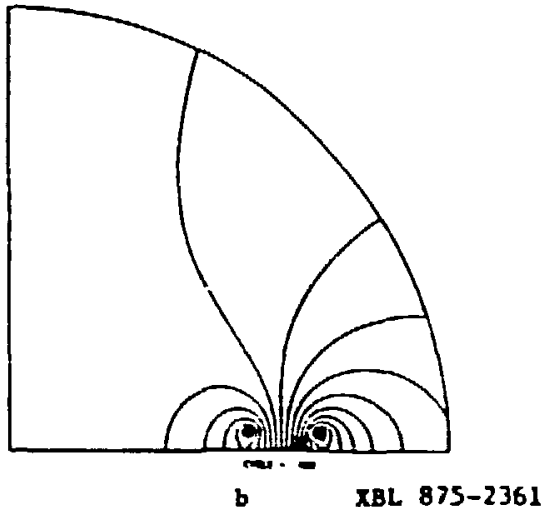

Fig. 3. Flux plot around a pair of conductors; (a) axiai symmetry with toroidal boundary, (b) axial symmetry with circular boundary (drawn to a reduced scale so as to include the axis of rotational symmetry). 
in Fig. 4. These variations include a comparison between two solutions tnat differ in the number of mesh points that have been used. (The cartesian case is a poor approximation and ls therefore omitted from Flg. 4.) Good agreement (<0.5\%) In $A^{\prime}$ ls obtalned between theory, clrcular b.c., and toroldal b.c.. The valuea for $B_{\text {r }}$ are compared In Flg. 5.

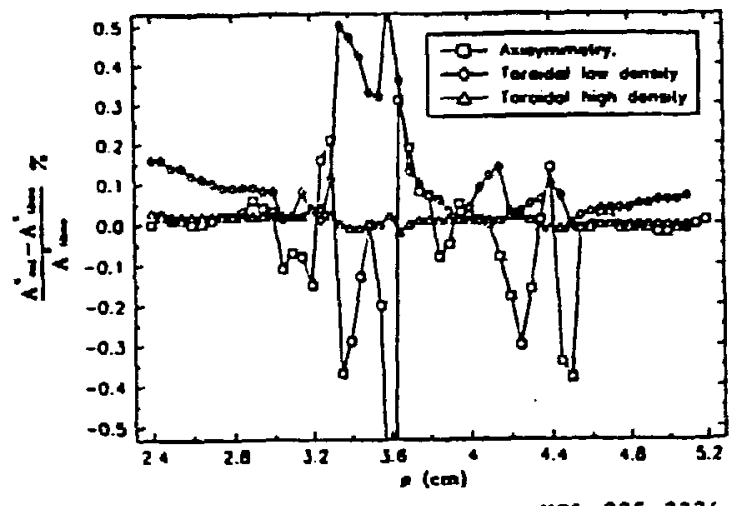

XBL $875-2374$

Fig. 4. Comparison between the calculated vector potential $A^{*}(=\rho A)$ and theoretical values along the midplane of symmetry $(z=0)$. The axisymmetric case Includes the axis of symmetry and employs a circular boundary, whereas the toroidal case employs a circular boundary around the sources. The need for a high mesh density is evident. It is noted that numerical difficultes will arise when $A$ approaches zero, causing fractional errors to be large. Such difficulties are present near $P_{1,0}$ for the toroidal case and exactly at $p_{3,0}$ for the cartesian case. In the data presented here, no attempt was made to overcome such difflculties and iarge fractional errors near $p_{1,0}$ accordingly do not reflect a real difference between the computated and expected values.

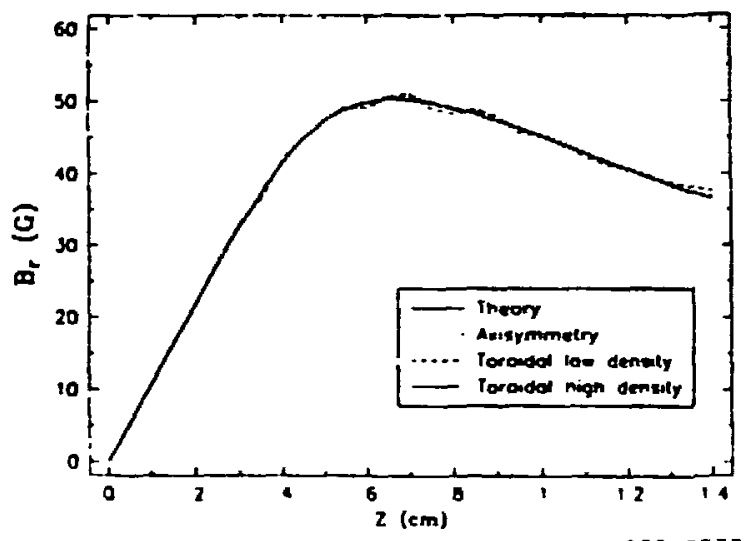

XBL $875-2373$

Fig. 5. The magnetic flux density in the $p$ direction along $p=3.75$ (note that $B_{t}$ for the Cartesian case is 0 ).

\section{Casse B - Coils Far From Axis}

The coils are now moved to $p_{1,0}=225 \mathrm{~cm}$ away from the axis of symmetry (Flg. 6). Cood agreement between the toroldal case and theary is maintained $\left(A^{*}<0.5 \%\right)$.

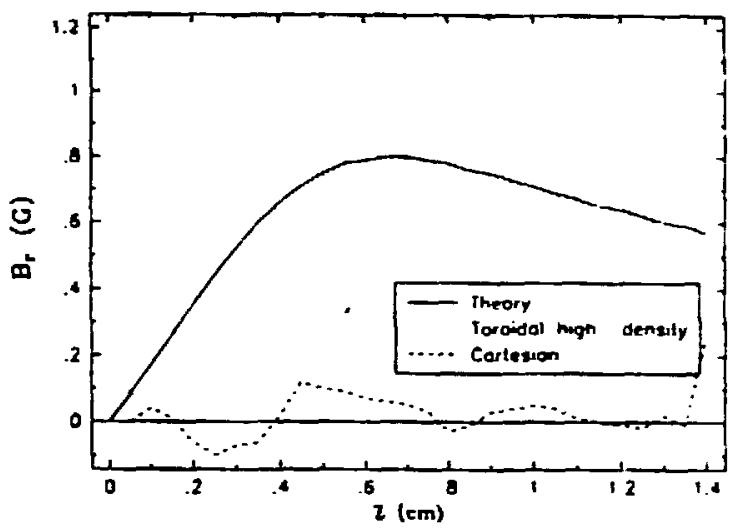

XBL $875-2369$

Fig. 6. Field values for a case where the coils have been extended to $\rho_{1,0}=225 \mathrm{~cm}$.

\section{REFERENCES}

1. L. Jackson Laslett, "On a Boundary Condition Applicable to Magnetostatic Relaxation Computations." ESCAR-28, November 1975.

2. L. Jackson Laslett and Victor O. Brady, "Incorporation of a Clircular Boundary Condition for a Magnet with Quadrant Symmetry Into the Program TRIM,LBID-172, January 1980.

3. 5. Caspl, M. Helm, and L. J. Laslett, "Incorporation of a Circular Boundary Condition into the Program POISSDN,- S5C-MAG-5/LBL-17064, March 2, 1984.

4. S. Caspi, M. Helm, and L. J. Laslett, The Ceneralization of a Circular Boundary Condition In the Program POISSON to Include No Symmetry and Axis-Symmetry of Revolution, SSC-MAG-12 LBL-I8063, July 6, 1984.

5. S. Caspi, M. Helm, and L. J. Laslett, "Incorporatlon of an Elliptical Boundary Condltion into the Program POISSDN,- 5SC-MAG-28/LBL-18798, December 1984.

6. 5. Caspl, M. Helm, and L. J. Laslett, "Incorporation of Superposition into the Program POISSON," SSC-MAG-31/RBL-19050, January 1985.

7. S. Caspi, M. Helm, and L. J. Laslett, -Numerical Solutlon of Boundary Condition to POISSON', Equation and Its Incorporation into the Program POISSON," Procezdings of the 1985 Particle Accelerator Conference, Vancouver BC, Canada, ssC-MAC-4II LBL-19483, May 1985.

8. 5. Caspi, M. Helm, and L. J. Laslett, "Incorporation ir Boundary Condition Into the Program PO:JuON," Proceedings of the 9th International Conference on Magnet Technology Zurich, Switzerland, SSC-MAG-sII LBL-19172, September 9-13, 1985.

9. L. J. Laslett, 5. Caspi, and M. Helm, -The Application of Program POISSON to Axially-Symmetric Problems with Use of a Prolate Spheroidal Boundary," SSC-MAC-68/LBL-20893, J anuary 1986.

10. L. J. Laslett, S. Caspi, and M. Helm, Incorporation of Toroidal Boundary Conditions Into Program POISSON, SSC-MAG-126/LBL-22985, July 17, 1987.

11. T. M. MacRobert, "Spherical Harmonics," Ed. 2, Dover, N.Y., 1947. 